\title{
The Combination of Moberg Flap with V-Y Advancement and Reverse Adipofascial Cross Finger Flap for Coverage of Degloving Injury of the Thumb-Case Report
}

\author{
Medhat Emil*, Dalia Habib, Omar Al Ameri, Dalal Al Mansoori \\ Department of Plastic and Reconstructive Surgery, Zayed Military Hospital, Abu Dhabi, UAE \\ Email: *medhatemil1@hotmail.com
}

How to cite this paper: Emil, M., Habib, D., Al Ameri, O. and Al Mansoori, D. (2020) The Combination of Moberg Flap with V-Y Advancement and Reverse Adipofascial Cross Finger Flap for Coverage of Degloving Injury of the Thumb-Case Report. Modern Plastic Surgery, 10, 56-61.

https://doi.org/10.4236/mps.2020.103007

Received: March 28, 2020

Accepted: May 6, 2020

Published: May 9, 2020

Copyright (c) 2020 by author(s) and Scientific Research Publishing Inc. This work is licensed under the Creative Commons Attribution International License (CC BY 4.0).

http://creativecommons.org/licenses/by/4.0/

Open Access

\begin{abstract}
We report a case of 22 years old male patient who is a worker in a factory and sustained degloving injury of his left thumb in a machine while working. There was loss of the pulp of the thumb extending circumferentially to the dorsal aspect with loss of the skin of the terminal phalanx and part of the proximal phalanx. The nail and germinal matrix were lost with exposure of the bone and extensor pollicis longus tendon insertion. The thumb was totally covered with a combination of two flaps: Moberg flap with V-Y advancement was used to cover most of the volar surface of the thumb and reverse adipofascial cross finger flap from the adjacent index finger was used to cover the dorsal surface and the tip of the thumb. The reverse adipofascial cross finger flap was covered with split thickness skin graft. Three weeks later this flap was divided and the thumb was mobilized freely. The patient had a full range of movement of the thumb and index finger with few settings of physiotherapy postoperatively. We recommend combining both of these flaps to reconstruct degloving injury of the thumb as they provide near adjacent tissue of similar texture, preserve sensation at the volar aspect of the thumb and also avoid the complications of the distant flaps.
\end{abstract}

\section{Keywords}

Moberg Flap, Reverse Adipofascial Cross Finger Flap, Degloving Injury

Thumb

\section{Introduction}

The thumb is responsible for about $40 \%$ of the function of the hand. Preserving 
every millimeter of it can make a difference in the patient's daily activities. Many techniques are described to reconstruct degloving injuries of the thumb. This article will discuss the procedure we used to reconstruct a degloving injury of the thumb using a combination of two flaps; one from the volar aspect of the thumb itself and another one from the adjacent index finger to preserve its length with durable flaps and preserve sensation at its volar aspect.

\section{Case Report}

A twenty two years old worker in a factory presented to the Emergency Department with circumferential degloving injury of his left thumb while working on a machine. There was loss of skin, subcutaneous fat, the whole nail and the nailbed and the tip of the head of the bone of the terminal phalanx (Figure 1). The bone of the terminal phalanx was totally exposed with exposure of the extensor tendon on the interphalangeal joint dorsally. The interphalangeal joint was covered with skin volarly with no exposure of the flexor pollicis longus tendon insertion. There was no skin at the dorsal aspect of the interphalangeal joint and the distal part of the proximal phalanx dorsally. The patient did not bring the degloved part with him as it was crushed in the machine.

\section{Operative Procedure}

The patient was taken to the operation theatre on the same day and under the effect of general anaesthesia and with the use of arm tourniquet, wound debridement was done. The volar skin was advanced distally using Moberg flap to cover most of the terminal phalanx (Figure 2). The flap is an axial one with both the digital arteries on the volar surface of the thumb included in it. To
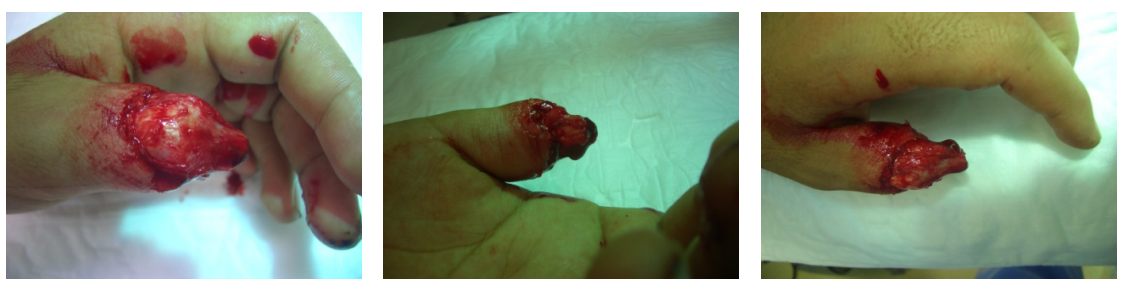

Figure 1. Circumferential degloving injury left thumb with loss of skin, subcutaneous fat, the whole nail and the nailbed and the tip of the head of the bone of the terminal phalanx. There is exposure of the bone of the terminal phalanx and the extensor tendon on the interphalangeal joint dorsally.
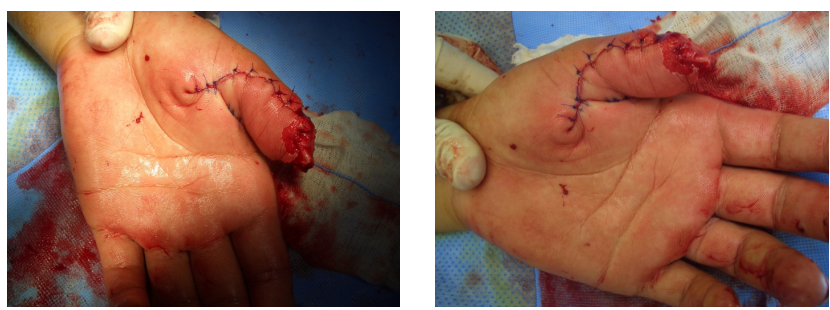

Figure 2. Moberg flap with V-Y advancement incorporation used to cover most of the terminal phalanx volarly. 
prevent flexion of the thumb at the interphalangeal joint, a " $\mathrm{V}$ " incision was made at the base of the thumb and was sutured in a "Y" shape fashion after advancing the flap distally as much as possible. Suturing done using 4/0 Prolene sutures. The flap could not reach the tip of the amputation stump and this part along with the dorsum of the thumb was covered with reverse adipofascial flap from the adjacent index finger.

The reverse adipofascial flap was designed on the dorsal aspect of the proximal phalanx of the left index finger. The skin (epidermis and dermis) was raised, like a page of a book with the base being on the contralateral side of the recipient digit (Figure 3). The adipofascial flap formed of all of the tissue between the dermis and paratenon, including the dorsal veins, fat and fascia. The flap was raised like a page of a book, with the base on the ipsilateral side of the thumb. Dissection of the flap was done with care to keep the paratenon of the extensor tendon intact. The adipofascial flap was inset to the defect and sutured to the radial edge and distal part of the Moberg flap using 4/0 Vicryl sutures. Split thickness skin graft was harvested from the medial aspect of the arm and applied on the flap after making few holes in the graft (Figure 4). The previously elevated skin of the proximal phalanx of the index finger was repositioned back to its original place to cover the paratenon of the extensor tendon and sutured to the surrounding skin proximally and distally with 5/0 Prolene sutures. It was sutured to the adjacent skin graft, but not to the underlying adipofascial flap, using 4/0 Vicryl sutures. A small tube drain was kept under the skin of the proximal phalanx of the index finger to prevent haematoma formation. Two $2 / 0$ Prolene sutures were used to keep the proximal part of the thumb and the index finger close together to avoid unexpected abduction of the thumb with resultant flap disruption during subsequent change of the dressings. Volar slab was applied in the functional position. The small tube drain was removed 2 days postoperatively. There was no haematoma formation and there was no infection. Both the flaps were viable and the graft take was $100 \%$. The reverse adipofascial flap was divided 3 weeks postoperatively and the patient started physiotherapy from the $2^{\text {nd }}$ day of the division (Figure 5). Physiotherapy and follow up continued for about 2 months and there was no stiffness of the thumb or the index finger.
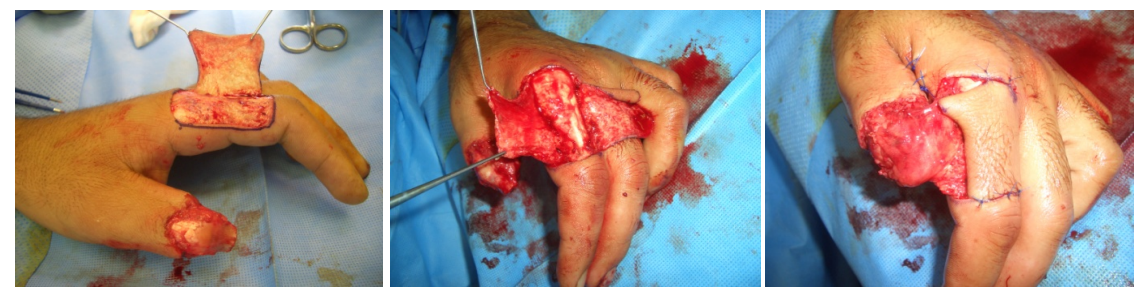

Figure 3. The reverse adipofascial flap: The skin (epidermis and dermis) was raised, like a page of a book with the base being on the contralateral side of the recipient digit. The adipofascial flap formed of all of the tissue between the dermis and paratenon raised like a page of a book, with the base on the ipsilateral side of the thumb. The paratenon of the extensor tendon is kept intact. The adipofascial flap was inset to the defect. 

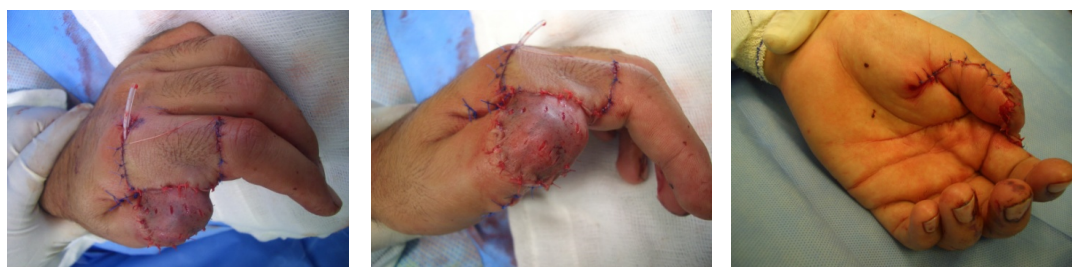

Figure 4. The adipofascial flap sutured to the radial edge and distal part of the Moberg flap and covered with split thickness skin graft. The previously elevated skin of the proximal phalanx of the index finger was repositioned back to its original place.
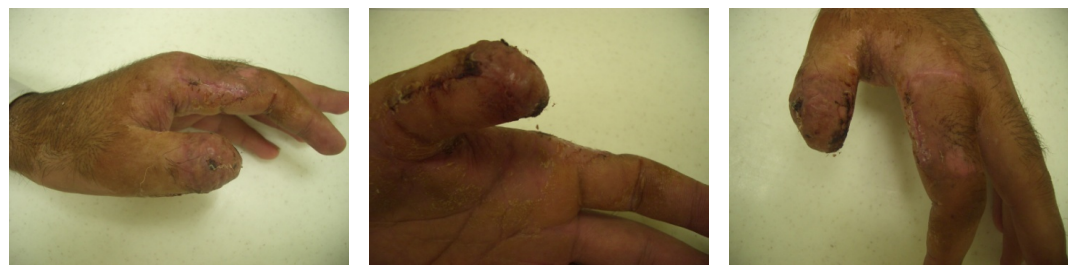

Figure 5. Three weeks later the reverse adipofascial flap was divided with good abduction of the thumb.

\section{Discussion}

The Moberg flap was first described in 1964 to cover defects at the pulp of the thumb by advancing the volar skin distally. It was used also with success in other fingers [1].

The Moberg flap involves making two bilateral incisions dorsal to the neurovascular bundles. The flap is raised over the paratenon including both the neurovascular bundles and advanced distally to cover the raw area at the tip of the thumb with the interphalangeal joint kept in flexion position. This can result in inability to extend the interphalangeal joint of the thumb fully post operatively [2]. To avoid this, different techniques were prescribed to provide more advancement of the flap without the need to keep the interphalangeal joint in flexion position. One of these techniques was prescribed by Jindal et al. in the form of " $Z$ " plasty modification at the base of the flap [3]. Another technique to avoid flexion of the interphalangeal joint is to incorporate " $V$ " at the base of the flap and the proximal defect is closed in a "V-Y" fashion [4]. In our case, we used the "V-Y" advancement technique to move the flap distally without flexing the interphalangeal joint of the thumb.

In a study done by Baumeister et al. on 36 patients, eighty three percent of the cases had the defects covered with the flap without additional iatrogenic shortening of the thumb [5]. In our case, the flap was advanced distally as much as possible but could not cover the whole distal phalanx volarly till the tip of the thumb. The bone was not shortened, as each millimeter of the thumb counts, but its tip was covered with the distal part of a reverse cross finger adipofascial flap from the dorsal aspect of the adjacent index finger. This flap was used also to cover the rest of the raw area at the dorsal aspect of the thumb.

Anatomic studies showed that this flap is based on constant dorsal branches of the palmar digital arteries in the proximal phalanx, which anastomose with the 
vascular system of the dorsal skin. The flap is drained by small vena concomitants that follow the arterial branches [6].

We elevated the flap based on this understanding of the blood supply after dissecting it from the overlying skin and the underlying paratenon of the extensor tendon of the index finger. The flap was turned over on its attached base at the radial side of the index finger to cover the raw area of the thumb and the skin over the donor site was repositioned over the paratenon. Split thickness skin graft was applied on the flap on the thumb only. We preferred this technique over other techniques as de-epithelialization of the skin of the donor finger and including it with the flap as one mass with turning over to cover the raw area [7] [8]. We feel that such procedures can predispose to implantation dermoid cyst formation if no adequate de-epithelialization of the flap done. Also applying skin graft on both the donor and recipient digits may be unnecessary if only skin graft can be applied on the recipient digit in the procedure we used avoiding disfigurement of the donor digit and maintaining its normal hairy skin shape.

Adipofascial flaps can also be used as turnover flaps from the same digit to cover defects of the digit distally. This homodigital flap can be used with success to cover the dorsal aspects of the middle [9] and terminal [10] phalanges of the fingers and also to cover raw areas at the dorsal aspect of the thumb [11]. It is a one stage procedure and does not involve other fingers than the one injured and when compared with the de-epithelialized cross finger flap it showed better results [12]. In our case we could not use this flap as the injury was circumferential and not involving the dorsal aspect only. Combining such a turnover flap with the Moberg flap can jeopardize the venous return of the thumb as the flaps are raised from both the volar and dorsal aspects of the thumb. Providing another flap from an adjacent finger can eliminate this risk.

\section{Conclusions}

The Moberg flap preserves the touch sensation at the volar aspect of the thumb replacing its pulp with the same type of tissue. Incorporation of V-Y advancement fashion at its base avoids flexion deformity of the thumb. The reverse adipofascial cross finger flap is a simple and rapid procedure. It is an excellent option because of its thinness, good pliability and minimal donor site deformity.

Combining both flaps together can avoid the need for distant flaps leaving the shoulder and hand entirely free and avoiding bulky disfigured insensate flaps.

\section{Conflicts of Interest}

The authors declare no conflicts of interest regarding the publication of this paper.

\section{Ethical Approval}

The procedures performed in this study involving human participant were in 
accordance with the international ethical standards.

\section{Informed Consent}

Additional informed consent was obtained from the participant for whom identifying information is included in this article.

\section{References}

[1] Macht, S.D. and Watson, H.K. (1980) The Moberg Volar Advancement Flap for Digital Reconstruction. Journal of Hand Surgery, 5, 372-376. https://doi.org/10.1016/S0363-5023(80)80179-1

[2] Ricardo, M. and Eilen, M. (2016) Moberg Advancement Flap in the Thumb. Orthopedics and Rheumatology Open Access Journal, 3, 555625.

https://doi.org/10.19080/OROAJ.2016.03.555625

[3] Jindal, R., Schultz, B.E., Ruane, E.J. and Spiess, A.M. (2016) Cadaveric Study of a Z-Plasty Modification to the Moberg Flap for Increased Advancement and Decreased Morbidity. Plastic and Reconstructive Surgery, 137, 897-904. https://doi.org/10.1097/01.prs.0000479988.80490.b8

[4] Page, F., Langley, C. and Lamyman, M. (2016) Management of Fingertip Injuries. The British Editorial Society of Bone \& Joint Surgery. Oxford University Hospitals NHS Foundation Trust Headley Way, Oxford.

[5] Baumeister, S., Menke, H., Wittemann, M. and Germann, G. (2002) Functional Outcome after the Moberg Advancement Flap in the Thumb. The Journal of Hand Surgery, 27, 105-114. https://doi.org/10.1053/jhsu.2002.30921

[6] Braga-Silva, J. (2005) Anatomic Basis of Dorsal Finger Skin Cover. Techniques in Hand and Upper Extremity Surgery, 9, 000-000. https://doi.org/10.1097/01.bth.0000173726.99670.f0

[7] Cappel, D.A. and Burech, J. (1985) The Cross-Finger Flap: An Established Reconstructive Procedure. Hand Clinics, 1, 677-683.

[8] Gürbüz, K., Uzun, E. and Ozan, F. (2016) Covering the Dorsal Finger Defect with Reverse Cross Finger Flap. Dorsal Defect and Cross Finger Flap. Journal of Clinical and Analytical Medicine, 7, 262-264. https://doi.org/10.4328/JCAM.4133

[9] Naji, S.H. (2011) Dorsal Adipofascial Turnover Flap for Dorsal Defects of Finger Injury. Medical Journal of Babylon, 8, 67-72.

[10] Ozdemir, R., Kilinç, H., Sensöz, O., Unlü, R.E. and Baran, C.N. (2001) Innervated Dorsal Adipofascial Turnover Flap for Fingertip Amputations. Annals of Plastic Surgery, 46, 9-14. https://doi.org/10.1097/00000637-200101000-00003

[11] Al-Qattan, M.M. (2001) The Adipofascial Turnover Flap for Coverage of the Exposed Distal Interphalangeal Joint of the Fingers and Interphalangeal Joint of the Thumb. Journal of Hand Surgery, 26, 1116-1119.

https://doi.org/10.1053/jhsu.2001.28758

[12] Al-Qattan, M.M. (2005) De-Epithelialized Cross-Finger Flaps versus Adipofascial Turnover Flaps for the Reconstruction of Small Complex Dorsal Digital Defects: A Comparative Analysis. The Journal of Hand Surgery, 30, 549-557.

https://doi.org/10.1016/j.jhsa.2005.02.004 